\title{
Consumo de drogas entre adolescentes: resultados de la Encuesta Nacional de Adicciones, 1998
}

\author{
Ma Elena Medina-Mora, D ra en Psic Soc, ${ }^{(1)}$ Patricia Cravioto, M en C, ${ }^{(2)}$ Jorge Villatoro, Psic, ${ }^{(1)}$ Clara Fleiz, Psic, ${ }^{(1)}$
} Fernando Galván-C astillo, Lic en Fís y Mat, ${ }^{(2)}$ Roberto Tapia-C onyer, MSP. ${ }^{(2)}$

\section{Medina-Mora ME, Cravioto P,Villatoro J, Fleiz C, Galván-Castillo F,Tapia-Conyer R. Consumo de drogas entre adolescentes: resultados de la Encuesta Nacional de Adicciones, 1998. Salud Publica Mex 2003;45 supl 1:S16-S25.}

\begin{abstract}
Resumen
Objetivo. Describir el consumo de drogas y los factores de riesgo en adolescentes de 12 a 17 años. Material y métodos. Los datos provienen de la Encuesta $\mathrm{N}$ acional de Adicciones 1998, realizada en hogares ubicados en zonas urbanas de la República Mexicana. El diseño de la muestra fue probabilístico, estratificado y por conglomerados en varias etapas de muestreo, donde la última unidad de selección fue un individuo en la vivienda. Los resultados se analizaron obteniendo los intervalos de confianza de las prevalencias del consumo de drogas, y se realizó un análisis de regresión logística para evaluar los factores asociados con el consumo de drogas. Resultados El $3.57 \%$ de los varones y $0.6 \%$ de las mujeres habían usado una 0 más drogas excluyendo al tabaco y al alcohol; 2.14 de hombres y $0.45 \%$ de mujeres lo habían hecho en los doce meses previos al estudio, y 1.4 de los hombres y $0.3 \%$ de las mujeres en los 30 días anteriores a la encuesta. La mariguana es la droga más usada (2.4 y $0.45 \%)$, seguida por los inhalables (1.08 y $0.20 \%$ ) y la cocaína $(0.99$ y $0.22 \%)$ por hombres y mujeres, respectivamente. El riesgo de usar drogas se asoció con ser hombre, no estudiar, considerar fácil conseguir drogas, no ver mal el uso de dro gas por parte de los amigos, que éstos las usaran, usarlas por parte de la familia y estar deprimido. Conclusiones El entorno que rodea a nuestros jóvenes de 12 a 17 años indica que el consumo de
\end{abstract}

\author{
Medina-Mora ME, Cravioto P,Villatoro J, Fleiz C, \\ Galván-Castillo F,Tapia-Conyer R. \\ Drug used among adolescents: \\ results from the National Survey \\ on Addictions, 1998.
}

Salud Publica Mex 2003;45 suppl 1:S16-S25.

\begin{abstract}
A bstract
Objective. The aim of this paper is to describe drug and associated factors use among adolescents (12 to 17 years of age). Material and Methods D ata come from the recent Encuesta $\mathrm{N}$ acional de Adicciones, 1998 (N ational Survey on Addictions) undertaken in urban areas of Mexico. A probabilistic, multi-stage, stratified cluster sampling design was used to select the study population. The sampling unit was the individual in each household. Statistical analysis consisted of the estimation of prevalences of drug use, with $95 \%$ confidence intervals. Associations were analyzed using logistic regressions models. Results. Excluding to bacco and alcohol, $3.57 \%$ of males and $0.6 \%$ of females have used one or more drugs; $2.14 \%$ and $0.45 \%$ used them in the 12 months previous to the survey, and $1.4 \%$ and $0.3 \%$ in the previous 30 days, respectively. Marihuana was the drug more often used by males $(2.4 \%)$ and females $(0.45 \%)$, followed by inhaled solvents $(1.08 \%$ and $0.20 \%)$, and cocaine $(0.99 \%$ and $0.22 \%$ ), respectively. The risk of using illicit drugs was associated to being male, having dropped out from school, perceiving availability, drug use by family and friends, social tolerance among friends, and symptoms of depression. Conclusions. A dolescents between 12 to 17 years of age are exposed to increasing drug use. The rates of drug use have risen, especially in the northern region of Mexico and in the big urban areas (Tijuana, Mexico City and Guadala-
\end{abstract}

(1) Instituto Nacional de Psiquiatría Ramón de la Fuente Muñiz, México, D.F., México.

(2) Secretaría de Salud, México, D.F., México.

Fecha de recibido: 18 de abril de 2001 • Fecha de aprobado: 11 de abril de 2002

Solicitud de sobretiros: Ma Elena Medina-Mora. Dirección de Investigaciones Epidemiológicas y Sociales, Instituto N acional de Psiquiatría Ramón de la Fuente Muñiz. Calzada México Xochimilco 101, 14370 México D.F., México. Correo electrónico: medinam@ imp.edu.mx 
dro gas está cada vez más presente. Los índices de consumo se han incrementado, especialmente en la región norte del país y en las grandes metrópolis (Tijuana, Ciudad de México y Guadalajara, principalmente). Estos datos refuerzan la necesidad de desarrollar campañas que permitan detectar a los menores que tienen problemas emocionales y darles la atención apropiada para evitar que consuman dro gas como un mecanismo para enfrentar problemas de esta naturaleza. A simismo, es necesario identificar a los menores con mayor probabilidad de desarrollar dependencia, quienes requieren de intervenciones preventivas más intensas.

Palabras clave: drogas ilícitas; ado lescencia; factores de riesgo; encuesta nacional; México jara). These findings emphasize the need to develop campaigns to detect emotional problems. Preventive measures should provide appropriate care to prevent drug use as a mechanism to cope with such emotional conflicts. Also, more intense preventive interventions should be targeted to adolescents with a high probability of developing drug abuse.

Key words: street drug; adolescence; risk factors; national survey; Mexico
E 1 consumo de drogas ha mostrado variaciones importantes a partir de la década de los 70, fecha en la que se realizan las primeras encuestas y otros estudios de carácter epidemiológico y social con el interés de conocer la distribución del consumo de drogas en el país. Al tradicional problema de uso de inhalables entre los menores, y de mariguana entre los jóvenes y algunos grupos de la población adulta, se suma un consumo cada vez mayor de cocaína que ha llegado hasta los niños y los sectores pobres de la población; aparecen nuevas drogas como las metanfetaminas y el inicio del consumo se ubica en edades cada vez más tempranas, de ahí que sea de interés estudiar al sector más joven de la población y dilucidar cuáles son los factores que los llevan al uso de drogas y, eventualmente, al uso problemático.

Las encuestas entre escolares realizadas en la década de los 70 indicaban que el abuso de inhalables era propio de las clases más desfavorecidas de la población, sin embargo, para 1984 el índice de usuarios de éstos mostraba ya cifras similares en escuelas ubicadas en zonas con diferentes niveles de servicios y de características de ingreso y empleo de sus habitantes. ${ }^{1}$ Después de un rápido incremento, a partir de 1980 los índices de inhalación se mantienen estables y es hasta los últimos años cuando el uso de estas sustancias parece estar disminuyendo ante el cambio de preferencias de la población usuaria por la cocaína. ${ }^{1-4}$

Esta tendencia se observa también en los sistemas de información disponibles; por ejemplo, el Sistema de Registro de la Ciudad de México (SRID) refleja un incremento en el número de usuarios de drogas que prefieren ahora consumir cocaína, quienes aumentaron de $4 \%$ de los casos en 1986, a 67\% en 1999..$^{5}$ La proporción de casos atendidos en centros de integración juvenil, para jóvenes que utilizan esta droga, aumentó 3.8 veces de 1990 a 1997, pasando de 12 a 46\% de los pacientes atendidos. El sistema de vigilancia epidemiológica de adicciones (Sisvea), reporta también un incremento en el número de usuarios de esta sustancia ilícita sin haber utilizado otra antes, o sin haber inhalado algún disolvente volátil; en 1991, 6\% de las personas que los habían usado, se había iniciado con ellos, proporción que sube a $16 \%$ en 1997.6

Aparecen en el mercado nuevas formas de cocaína como el crack, presentación más adictiva y dirigida a personas de menos recursos económicos. En la frontera norte del país aumenta en forma importante el consumo de heroína, cuyo uso por inyección intravenosa se combina más frecuentemente con cocaína.*

El uso de drogas del tipo de las metanfetaminas empieza a documentarse en los primeros años de esta década; su consumo es más frecuente en la frontera noroccidental del país. El sistema de registro de pacientes de centros de integración juvenil señala una media nacional de $2.7 \%$ para 1995, año en el que los había consumido $42 \%$ de los pacientes atendidos en el centro ubicado en la Ciudad de Tijuana, Baja California. $^{7}$

En el contexto internacional, México se ubica entre aquellos países que tienen bajas tasas de consumo, pero que, a su vez, reportan incremento del problema. ${ }^{8}$ La Organización de las Naciones Unidas ${ }^{8}$ estima una prevalencia mundial de $4.2 \%$ para cualquier droga

\footnotetext{
* Cravioto P, Medina-Mora ME, De la Rosa B, Galván F. El problema de la heroína en Ciudad Juárez, Chihuahua. Protocolo de investigación en proceso. México, D.F.: Dirección General de Epidemiología, Instituto Nacional de Psiquiatría, 2000.
} 
ilícita en la población de 15 años en adelante, en tanto que en México el índice para la población de 12 a 65 años es de $1.2 \% .{ }^{9}$ Los índices para mariguana son, entre hombres y mujeres de 3.4 y $1 \%$, respectivamente, para heroína $0.22 \%$ y $<0.1 \%$, mientras que para la cocaína, el consumo en México es superior al promedio mundial: $0.3 \%$ y $0.5 \%$. Esta situación se deriva de su amplia disponibilidad en la región; se ha estimado que 98\% de la producción mundial se da en la Región Andina y 70\% del consumo mundial ocurre en América. Dentro de la Región, México presenta las tasas más bajas de consumo; el índice en Estados Unidos de América es seis veces superior (3.0\%). En Argentina alcanza 2.3\%, en Chile 2.1\%, en Colombia 1.2\%, en Perú $1 \%$ y en Brasil, $0.8 \%{ }^{8}$ El índice de dependencia reportado en México (0.7\%), es también inferior al observado en Estados Unidos de América (7.5\%) y en países europeos como Alemania $(2.1 \%)$ y Holanda $(1.8 \%) .{ }^{10}$

Este artículo describe el índice de experimentación y consumo en la población adolescente de entre 12 a 17 años que habita en zonas urbanas del país de más de 2500 habitantes; se proporcionan prevalencias de uso de alguna vez de los últimos 12 meses y de los 30 días previos a la encuesta, los problemas asociados con el consumo, y se muestran las variaciones regionales. Se comparan los índices en población matriculada y no matriculada a la escuela, se describen las variaciones para el uso de cualquier droga (mariguana, inhalables, alucinógenos, cocaína, medicamentos depresores y estimulantes tomados sin prescripción médica) agrupados por sexo, edad y regiones del país, y se describen a partir de modelos de regresión logística las circunstancias que actúan como factores de riesgo y protección para el uso de drogas ilícitas.

\section{Material y métodos}

La tercera Encuesta Nacional de Adicciones (Enadic 1998) se realizó con el fin de actualizar la información sobre la prevalencia y abuso de sustancias adictivas entre los residentes habituales de viviendas particulares, adultos entre 18 y 65 años, y adolescentes entre 12 y 17 años de edad. Este artículo sólo contiene la información para población adolescente.

Se utilizó un cuestionario estandarizado ya empleado en estudios previos y completado en una entrevista individual. ${ }^{11,12}$ La sección sobre consumo de sustancias y problemas fue probada por diferentes países, entre ellos México, conjuntamente con la Organización Mundial de la Salud ${ }^{13}$ y aplicada en encuestas a estudiantes y en hogares desde 1974..$^{1-4,14-16}$ Mediante estas preguntas es posible determinar la prevalencia global (uso alguna vez), la prevalencia lápsica (uso en los 12 meses previos a la encuesta) y prevalencia actual (uso en los 30 días anteriores). A partir de estos indicadores se calcula la tasa de continuidad para hombres y mujeres por cada tipo de droga (se refiere al porcentaje de adolescentes que habiendo usado la droga alguna vez, reconocieron seguir usándola en los 12 meses o 30 días previos al estudio).

En el cuestionario se incluyeron cuatro preguntas que evalúan problemas con drogas: haber estado hospitalizado por el uso de drogas, haber sido arrestado o asaltado después de consumirlas y el deseo de consumir menos drogas. Contiene escalas que evalúan: a) percepción de disponibilidad, para averiguar qué tan fácil o difícil sería para el adolescente conseguir diferentes tipos de drogas si quisiera, ${ }^{17,18} \mathrm{~b}$ ) tolerancia social y percepción de riesgo, evaluadas mediante escalas adaptadas de las encuestas nacionales de población estudiantil realizadas en Estados Unidos de América por el Institute for Social Research ${ }^{19} \mathrm{y}$ utilizadas en México desde la década de los 70; c) consumo de drogas en la familia y por amigos, lo cual se evalúa mediante preguntas elaboradas ex profeso para encuestas estudiantiles; ${ }^{3}$ d) la depresión, se midió por medio de la Escala de Depresión del Centro de Estudios Epidemiológicos (CES-D) ${ }^{20}$ para adolescentes, probada en México; ${ }^{21}$ consta de 20 reactivos calificados de 0 a $4(0=$ no ha presentado el síntoma ningún día, $1=$ ha presentado el síntoma de 1 a 2 días, $2=$ ha presentado el síntoma de 3 a 4 días, $3=$ ha presentado el síntoma de 5 a 7 días) y el punto de corte para un posible caso es con una puntuación de 16 o más, y e) ideación suicida e intento de suicidio, se evalúa mediante tres reactivos de la escala de Roberts ${ }^{17}$ y de preguntas elaboradas ex profeso por González-Forteza, ${ }^{22}$ para diferentes encuestas realizadas con estudiantes de nivel medio.

La relación entre variables y el uso de drogas se analizó mediante regresión logística; se tomó el uso de cualquier droga ilegal sin hacer distinciones por su tipo, debido al reducido tamaño de la muestra. Las variables que intervinieron en el análisis fueron tratadas como dicotomías, se dio valor 1 a ser varón y 0 a ser mujer; un valor 1 al grupo entre 12 y 15 años, y de 2 a aquel entre 16 y 17 años. Un valor de 1 se dio a estudiar y 2 a no estudiar. También recibió un valor 1 trabajar y establecer una escolaridad superior a primaria del jefe de familia. En tanto que no trabajar y tener un jefe de familia con una escolaridad inferior recibieron un valor de 0 . El consumo entre padre, madre, hermanos, familiares y mejor amigo recibió también una calificación de 1 , y de 0 cuando estas situaciones estaban ausentes; se dio también un valor 1 cuando el adolescente afirmó que era fácil o muy fácil conseguir 
drogas, cuando consideraba que sus padres o sus amigos verían bien, o les daba igual, que él las consumiera, cuando consideraban que no era peligroso usarlas, cuando tenían una calificación superior a 16 puntos en la escala de depresión y de cero en los otros casos. Finalmente, la ideación suicida tuvo valor de 0 a 4, dependiendo del número de síntomas presentes.

Con objeto de conocer los factores que se relacionan con la experimentación (es decir aquellos adolescentes que han consumido cualquier droga entre una y cinco veces) y con el uso continuado de drogas (se refiere a aquellos adolescentes que han consumido seis o más veces), se realizó una serie de regresiones logísticas. Específicamente se realizaron tres análisis cambiando los valores de la variable dependiente. En el primero, se tomó a no usuarios-experimentadores; en el segundo, no-usuarios-uso continuado, y en el tercero, uso experimental-uso continuado. Finalmente, se compararon aquellos adolescentes usuarios de drogas que reportaron problemas en comparación con los que no lo hicieron. Este análisis incluyó solamente a aquellos adolescentes que habían usado drogas ilícitas (mariguana, cocaína, alucinógenos e inhalables). Los análisis se obtuvieron con el paquete SPSS, versión 8.0.

El diseño de la muestra ha sido publicado en otros informes, ${ }^{19,23}$ basta mencionar que fue probabilístico estratificado con selección de conglomerados en varias etapas de muestreo (Area Geoestadística BásicaAGEB), manzanas, viviendas e individuo. Para ello, se usó el marco cartográfico y de datos en nivel localidad y AGEB del Conteo Nacional de Población de 1995, realizado por el Instituto Nacional de Estadística, Geografía e Informática (INEGI).

Se crearon nueve estratos geográficos: las tres áreas metropolitanas más grandes del país, cuya delimitación se basó en la definida por el INEGI para el Conteo de Población 1995, tres ciudades fronterizas y tres regiones del país.

La muestra fue calculada con una precisión de 3\%, un nivel de confianza de $95 \%$ para los datos nacionales; para los nueve estratos, la precisión fue de $5 \%$, con una confianza de entre 90 y $95 \%$. El efecto de diseño fue de 2.3 a 3.5 para valores de parámetros que oscilaron entre 20 y $50 \%$. Se seleccionaron 4325 adolescentes de los cuales se obtuvieron 3882 entrevistas completas y 443 entrevistas fueron registradas como no respuesta, equivalentes a $9.6 \%$, ya que entre diferentes razones, en $3.4 \%$ no se encontró a ninguna persona en casa y $3.3 \%$ se negó a dar información. La tasa de no respuesta de la encuesta tuvo un comportamiento diferencial por estrato, por ello se aplicó un factor de corrección a las estimaciones cuando se calcularon és- tas para el conjunto de los estratos. Los encuestadores fueron capacitados en los aspectos operativos del trabajo de campo y en el manejo del cuestionario.

\section{Resultados}

Las características demográficas de los usuarios se muestran en el cuadro I.

\section{Indices de consumo}

Los índices de consumo de sustancias en esta temprana edad son bajos; entre los varones oscilan de $2.5 \%$, en el caso de la mariguana, a $0.2 \%$ en el de alucinógenos; entre las mujeres los índices fueron aún menores, oscilando entre 0.5 y $0.1 \%$ para estas mismas dos sustancias (cuadro II). Sin considerar a los alucinógenos

\section{Cuadro I \\ Características sociodemográficas DE ADOLESCENTES DE 12 A 17 AÑOS. Encuesta Nacional de Adicciones 1998. México}

\begin{tabular}{lcc}
\multicolumn{1}{l}{$\begin{array}{c}\text { Hombres } \\
\%\end{array}$} & $\begin{array}{c}\text { Mujeres } \\
\%\end{array}$ \\
Edad & & \\
12 a 14 & 53 & 50.5 \\
\hline 15 a 17 & 47 & 49.5
\end{tabular}

Escolaridad

\begin{tabular}{lll} 
Primaria & 32 & 31 \\
\hline Secundaria o equivalente & 51 & 51 \\
\hline Preparatoria o equivalente & 16 & 18
\end{tabular}

Estatus educativo

\begin{tabular}{lll} 
Estudia & 81 & 79 \\
\hline No estudia & 19 & 21
\end{tabular}

Razones de abandono de la escuela

\begin{tabular}{lcc} 
Problemas económicos & 35 & 37 \\
\hline Problemas escolares & 18 & 5 \\
\hline De salud & 6 & 3 \\
\hline Embarazo & - & 6 \\
\hline Personales & 17 & 18 \\
\hline No quiso seguir estudiando & 23 & 25 \\
\hline U so de drogas o alcohol & 1 & -
\end{tabular}

0 cupación

\begin{tabular}{lrc} 
Sólo estudia & 74 & 77 \\
\hline Estudia y trabaja & 7 & 2.3 \\
\hline Sólo trabaja & 19 & 16 \\
\hline
\end{tabular}


Cuadro II

Prevalencia de uso de drogas entre adolescentes de 12 a 17 años. Encuesta Nacional de Adicciones 1998, México

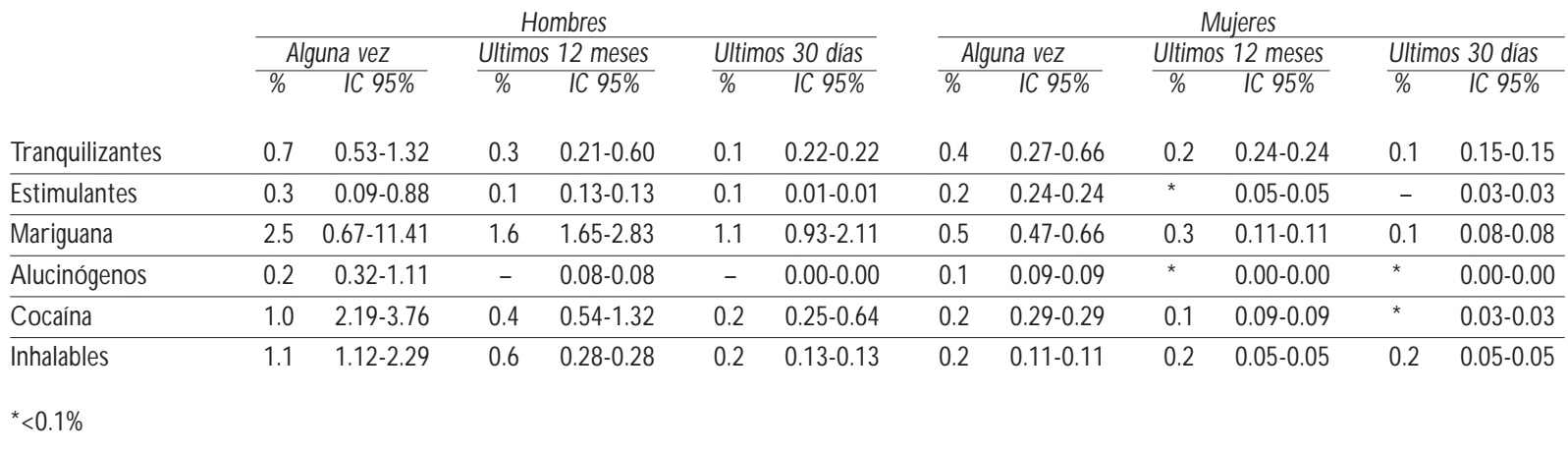

que mostraron índices de consumo muy bajos, alrededor de la mitad de los menores que reportan uso de drogas alguna vez, continuaba haciéndolo en los 12 meses previos al estudio.

En total, $1.3 \%$ de las mujeres y $3.4 \%$ de los varones reportaron haber experimentado entre una y cinco veces una o más de las drogas consideradas; 0.3 y 1.5\% habían continuado su uso después de experimentar los efectos. En ambos grupos, los alucinógenos tienden a ser sustancias de experimentación, en tanto que la mariguana y los estimulantes en los varones, y los estimulantes y la cocaína entre las mujeres, son las drogas que con más frecuencia se continúan usando después de experimentar con sus efectos (cuadro III).

Con el objeto de verificar la veracidad de las respuestas dadas por adolescentes, el cuestionario incluyó preguntas que investigaban el uso de sustancias ficticias; en esta encuesta, no se reportó consumo de ninguna de estas sustancias.

Ocho de cada 100 varones y cinco de cada 100 mujeres que han usado drogas reportaron haber estado hospitalizados por razones relacionadas con el uso, $11 \%$ de los varones y $2 \%$ de las mujeres reportaron haber sido arrestados por la policía, 6 y $0.5 \%$ reportaron haber sido asaltados después de haber estado consumiendo, y $41 \%$ de los varones y $28 \%$ de las mujeres reportaron desear consumir menos drogas. En total $28 \%$ de las mujeres y $45 \%$ de los varones que habían usado drogas, manifestaron tener uno o más problemas derivados de su consumo.

Existen importantes variaciones en los índices de consumo de sustancias en las diferentes regiones del país, con índices más altos en la zona norte (5.6\% entre los varones y $1.5 \%$ en las mujeres) en comparación con las zonas centro $(4.7$ y $2.1 \%)$ y sur $(4.4$ y $0.9 \%)$. En- tre las ciudades estudiadas, Tijuana, Ciudad Juárez, Guadalajara y Monterrey, muestran los índices más elevados de consumo, la Ciudad de México se caracteriza por su elevado consumo en las mujeres (cuadro IV).

\section{Factores asociados con la iniciación}

Los inhalables son las sustancias de inicio más temprano, seguidos por la mariguana; el consumo de co-

\section{Cuadro III \\ TASA De CONTINUIDAd EN EL USO DE DROGAS ILÍCITAS, Y TIPO DE USUARIO ENTRE ADOLESCENTES DE 12 a 17 aÑos. EnCUesta NaCIONAL de Adicciones 1998, México}

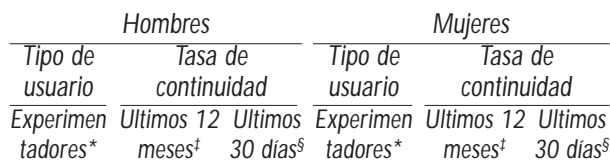

\begin{tabular}{lrrrrrr} 
Tranquilizantes & 80 & 51 & 36 & 77 & 55 & 63 \\
\hline Estimulantes & 48 & 56 & 38 & 59 & 10 & 0 \\
\hline Mariguana & 61 & 63 & 71 & 93 & 60 & 38 \\
\hline Alucinógenos & 85 & 0 & 0 & 100 & 27 & - \\
\hline Cocaína & 72 & 45 & 35 & 41 & 58 & 43 \\
\hline Inhalables & 74 & 54 & 31 & 96 & 96 & 96
\end{tabular}

* Porcentaje del total de usuarios que reportaron haber experimentado con cada droga de una a cinco veces; la diferencia a 100\% se refiere a menores que continuaron el uso después de experimentar con drogas

₹ Porcentaje que habiendo usado la droga alguna vez, reportó seguir usándola en los 12 meses previos al estudio

$\S$ Porcentaje que habiendo usado la droga en los últimos 12 meses, reportó seguir usándola en los 30 días previos al estudio 


\section{Cuadro IV \\ Variaciones en el consumo de dRogas alguna vez POR REGIONES DEL PAís PARA CADA SEXO, ENTRE adolescentes de 12 a 17 años. Encuesta Nacional de Adicciones 1998, México}

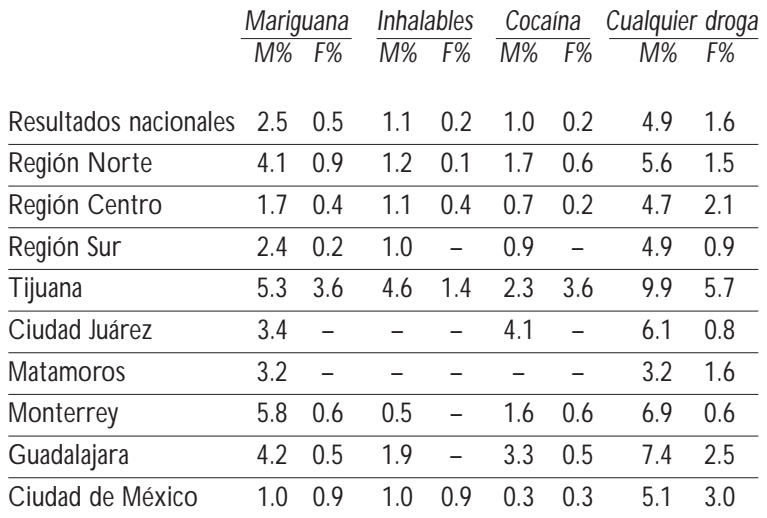

caína se inicia a partir de los 11 o 12 años, sin embargo, cuando adolescentes inician el uso después de los 14 años, es más probable que lo hagan con mariguana o cocaína que con sustancias inhalables.

Las calles o los parques son los lugares en donde con más frecuencia se obtienen las diferentes drogas; cerca de la mitad de los usuarios de mariguana (49\%) y de alucinógenos (47\%), y cerca de una tercera parte de aquellos que reconocieron haber usado inhalables (38\%) y cocaína (31\%) adquirieron las drogas en estos lugares; las casas particulares son lugares usuales para la adquisición de estas dos últimas drogas (32 y 29\%); las fiestas son lugares comunes para la mariguana $(25 \%)$ y para la cocaína ( $23 \%)$, en tanto que las discotecas sólo fueron lugares importantes para la adquisición de esta última sustancia $(12 \%)$.

Las razones más importantes en los varones para usar drogas fueron ver si les gustaba (27\%); porque les dijeron que se sentía bien (22\%) o porque sus amigos las estaban usando (16\%); entre las mujeres, que se sentía bien fue la razón más importante $(26 \%)$, en segundo lugar dijeron haber probado la droga para ver si las tranquilizaba (20\%) y en tercer lugar para ver si les gustaba $(14 \%)$. Doce de cada 100 varones respondieron que usaron la droga para ver si los tranquilizaba y 10 de cada 100 mujeres, porque sus amigos las estaban usando. El 7\% de los varones reportó haberlas usado por no tener otra cosa que hacer; esta razón no fue proporcionada por las mujeres de la muestra.

\section{Factores con los que se asocia el consumo}

\author{
Disponibilidad y uso de sustancias
}

El 35\% de adolescentes informaron que les era fácil obtener drogas, a $21 \%$ de los varones y a $10 \%$ de las mujeres de hecho se las habían ofrecido. El $67 \%$ de los varones y $57 \%$ de las mujeres que habían usado drogas dijo que un amigo se las había proporcionado por primera vez, $18 \%$ de los varones y $40 \%$ de las mujeres habían obtenido la droga por primera vez de un familiar, 6 y $4 \%$ respectivamente, un compañero de la escuela, y solamente $5 \%$ de los varones, y ninguna mujer, reportó que la habían obtenido de un vendedor.

\section{Tolerancia social y uso de sustancias}

En general existe poca tolerancia social para el consumo de drogas, más de $90 \%$ de los varones y de las mujeres afirmaron que sus papás, y 83 y $91 \%$ que sus amigos verían mal si ellos o ellas usaran drogas; la percepción sobre la aceptación del consumo entre los jóvenes es mayor, 38\% de los varones y la misma proporción de mujeres consideraron que los jóvenes en general ven bien o, con indiferencia, el consumo. Sin embargo, al analizar la relación entre la percepción de aceptación y el consumo de drogas se encuentran diferencias interesantes, siendo más frecuente el uso cuando el menor percibe aceptación social hacia el consumo; solamente $3 \%$ de quienes perciben poca tolerancia en sus padres aceptaron consumir drogas, en comparación con $14 \%$ de quienes perciben tolerancia; la misma tendencia se observa en relación con la tolerancia por parte de amigos cercanos, 1.8 y $5.2 \%$ entre hombres y mujeres, respectivamente.

\section{Consumo en el entorno inmediato y el uso de drogas}

El 1.8\% de adolescentes varones confirmaron que su papá consumía drogas, para $0.2 \%$ era la madre, y 2.1\% reconoció a algún hermano como consumidor. Las mujeres reportaron índices similares de exposición a las drogas en su medio inmediato; los índices entre éstas fueron 1.1, 0.5 y 2.4\%, respectivamente. Cuando esto ocurre es más probable que el menor experimente con drogas, así es más probable que un menor pruebe las drogas cuando sabe que su padre las usa y que continúe usándolas después de haber experimentado con ellas. Cuando es la madre la que abusa de sustancias, el riesgo de que el menor experimente tam- 
bién aumenta. Los hermanos son la influencia más poderosa, $15.7 \%$ de los menores cuyos hermanos eran usuarios de drogas las habían experimentado y $8.8 \%$ continuaba el uso, en comparación con solamente 2 y $0.7 \%$ de aquellos que no tenían hermanos usuarios. El uso entre amigos es también un predictor importante del consumo, $12.7 \%$ de los varones y $9.3 \%$ de las mujeres reportaron que sus amigos usaban sustancias; 9.4\% de aquellos cuyo mejor amigo usaba drogas había a su vez experimentado con ellas, en comparación con solamente $1.5 \%$ de aquellos cuyo mejor amigo no las usaba.

Percepción de riesgo y uso de sustancias

En general los jóvenes perciben que es riesgoso usar sustancias, $76 \%$ de los varones y $80 \%$ de las mujeres indicaron que consumir mariguana o cocaína era peligroso. Las razones más importantes que aducían los no usuarios por no haber usado drogas fueron el considerar que podía ser dañino para la salud (40 y $43 \%$ ) y el que no les interesaba ( 28 y $32 \%$ ); el temor a tener problemas con los padres ocupó el tercer lugar (11 y 9\%), en tanto que la percepción de ilegalidad (0.5 y $0.2 \%$ ) no es un factor importante.

La percepción de riesgo es un factor que afecta poco la decisión del adolescente de experimentar con drogas; $2.6 \%$ de los experimentadores consideraron que usar drogas era peligroso, en comparación con $2.6 \%$ que aseveró que no lo era. Sin embargo, esta variable es importante para detener el uso de los menores después de haber experimentado; aquellos que consideraron que usar drogas es muy peligroso presentaron frecuencias de consumo 3.8 veces inferiores.

\section{Estudio, trabajo y uso de drogas}

La asistencia a la escuela sigue teniendo una influencia considerable en las prácticas de consumo; mientras que $5.9 \%$ de los menores que no asistían a la escuela habían consumido una o más drogas, esto ocurría solamente en $2.2 \%$ de aquellos que asistían a la escuela. De manera similar $84 \%$ de los varones y $69 \%$ de las mujeres que habían usado drogas tenían un mal desempeño escolar. Sin embargo, la asistencia a la escuela es sólo un factor protector para los varones; ya que de los que asisten sólo $2.9 \%$ consumen drogas, en tanto que los que ya no asisten consumen en $11.4 \%$. En comparación con las mujeres, de las que estudian 1.3\% consume drogas, y el porcentaje es muy similar para quienes ya no estudian: $0.8 \%$. Los menores que trabajan y que perciben un salario experimentaron con más frecuencia con drogas, $5.2 \%$, en comparación con los que no habían trabajado (1.8\%); de manera similar, 1.3 y $0.8 \%$ habían usado drogas en más de cinco ocasiones.

Problemas emocionales y uso de sustancias

Uno de cada cinco adolescentes encuestados informaron de síntomas de depresión, aunque el uso de drogas y la escala de sintomatología depresiva se midieron por separado, se observó que la presencia de esta sintomatología se asoció con la experimentación con drogas, así 5.2\% de aquellos por arriba del punto de corte de la escala reportaron haber experimentado con sustancias, en comparación con solamente $1.6 \%$ de aquellos que no presentaban síntomas de malestar emocional de tipo depresivo. Quienes presentaron estos síntomas con más frecuencia continuaron usando las drogas una vez iniciado el consumo. El 1.34\% de adolescentes entrevistados dijeron haber intentado suicidarse; este índice era cinco veces más elevado entre quienes usaban drogas y tuvo una incidencia cinco veces mayor entre quienes reportaron tener problemas con el alcohol o las drogas.

\section{Predicciones de la experimentación, uso continuado y problemas con las drogas}

No uso vs uso experimental

El análisis de regresión logística mostró que el ser varón aumentó 2.87 veces el riesgo de usar drogas y el no estudiar 2.06 veces. La edad, el haber trabajado o la escolaridad del jefe de familia no resultaron predictores significativos para diferenciar a aquellos jóvenes que no usan drogas, de aquellos que deciden experimentar. La percepción de riesgo o la tolerancia social en la familia no modificaron la decisión del individuo, sin embargo, el riesgo de experimentar se incrementa 1.89 veces cuando les es fácil conseguir drogas, 1.69 veces cuando el mejor amigo no desaprobaba el consumo, 2.69 veces cuando los amigos las usaban y 3.78 veces cuando era un familiar el que las usaba. Finalmente, el que el joven experimentara problemas emocionales que denotaran depresión fue un factor importante y cuya presencia incrementó 3.22 veces el riesgo de experimentar con drogas (cuadro V).

No uso vs uso continuado

Cuando se comparan las poblaciones de no usuarios con las de usuarios de drogas se encuentra que la percepción de disponibilidad (2.80 con intervalo de 1.12-7.00), el consumo de drogas en la familia (7.60; 


\section{Cuadro V \\ FACTORES QUE DIFERENCIAN A LOS NO USUARIOS DE LOS EXPERIMENTADORES DE DROGAS ILÍCITAS ENTRE ADOLESCENTES DE 12 A 17 AÑOS DE EDAD. ENCUESTA Nacional de Adicciones 1998. México}

\begin{tabular}{lllll}
$\begin{array}{l}\text { No usuario-usuario } \\
\text { experimental }\end{array}$ & $\mathrm{P}$ & $\mathrm{R}$ & $\operatorname{Exp}(\mathrm{B})$ & $\mathrm{IC} 95 \%$ \\
Edad del sujeto & 0.06 & 0.04 & 1.57 & $0.97-2.51$ \\
\hline Estatus escolar & 0.01 & 0.08 & 2.06 & $1.22-3.50$ \\
\hline Estatus laboral & 0.23 & 0.00 & 1.39 & $0.82-2.35$ \\
\hline Escolaridad del jefe de familia & 0.23 & 0.00 & 0.75 & $0.47-1.19$ \\
\hline Disponibilidad & 0.01 & 0.07 & 1.89 & $1.17-3.05$ \\
\hline Tolerancia social familia & 0.66 & 0.00 & 0.72 & $0.17-3.06$ \\
\hline Tolerancia social pares & 0.03 & 0.06 & 1.69 & $1.05-2.74$ \\
\hline Consumo de drogas familia & 0.00 & 0.12 & 3.78 & $1.92-7.42$ \\
\hline Consumo de drogas amigos & 0.00 & 0.13 & 2.69 & $1.64-4.42$ \\
\hline Percepción de riesgo & 0.97 & 0.00 & 1.01 & $0.55-1.87$ \\
\hline Depresión & 0.00 & 0.15 & 3.22 & $1.93-5.38$ \\
\hline Ideación suicida & 0.82 & 0.00 & 0.97 & $0.74-1.26$ \\
\hline Sexo & 0.00 & 0.13 & 2.87 & $1.75-4.72$ \\
\hline Constante & 0.00 & & &
\end{tabular}

2.73-21.15), el consumo de drogas entre los amigos $(5.89 ; 2.50-13.85)$, la alta percepción de riesgo (4.83; 2.11-11.06) y el ser varón (10.24; 3.4-30.86) diferencian a aquellos adolescentes que no usan drogas de aquellos que continúan usándolas después de haber experimentado sus efectos.

\section{Uso experimental vs uso continuado}

El paso de la experimentación al uso continuado estuvo determinado por la percepción de que los padres no verían mal el uso (8.04; 1.16-55.74), el que adolescentes no percibieran que usar drogas fuera peligroso $(3.95 ; 1.38-11.29)$ y el que fueran varones $(5.54 ; 1.27-$ 24.10).

\section{Uso con problemas vs uso sin problemas}

La única variable que resultó significativa para diferenciar a aquellos adolescentes que reportaron nunca haber estado hospitalizados por el uso de drogas, no haber sido arrestados ni asaltados después de haber estado consumiendo o que no deseaban consumir menos drogas, de aquellos que reportaron al menos uno de estos problemas, fue el no percibir riesgo asociado con el consumo de drogas (3.81; 1.43-10.16).

\section{Discusión}

Los datos nos indican que las drogas están presentes en la población adolescente entre 12 y 17 años de edad, lo cual se manifiesta en las zonas urbanas de más de 2500 habitantes en todo el país. Si bien los índices de consumo son mayores en la zona norte y en las grandes metrópolis, una parte importante de las diferencias con las ciudades y regiones con índices más bajos se debe en cierta medida a un menor uso entre mujeres, pues éstas siguen consumiendo en menor proporción que los varones.

Como se esperaba, debido a la evidencia proveniente de otros estudios ${ }^{(4-6,7)}$ la cocaína ha ocupado un lugar preponderante en las preferencias de los adolescentes y cuando no han probado drogas antes de los quince años, y deciden experimentar, es probable que lo hagan con cocaína.

Al comparar los resultados de esta encuesta ${ }^{9}$ con las dos Encuestas Nacionales de Adicciones anteriores $^{16,24}$ y que abarcan un periodo de 10 años, se hacen evidentes considerables aumentos en la prevalencia nacional del consumo alguna vez de drogas ilícitas, tendencia que se había perfilado desde la Encuesta Nacional de Adicciones de 1993. De esta forma, el consumo de mariguana se elevó significativamente entre las dos últimas encuestas; mientras en 1988 el consumo encontrado fue de $2.99 \%$, en 1998 se elevó a $4.70 \%$. La proporción de los que usaron inhalables alguna vez había ido descendiendo ligeramente entre 1988 y 1993 (0.76 y 0.50\%, respectivamente); sin embargo, en 1998 se observó un ligero incremento $(0.80 \%)$. La cocaína es la droga que registra los incrementos más importantes, al casi triplicarse el consumo alguna vez en $1998(0.33 \%)$, respecto a 1988 (1.45\%). El uso de heroína casi no ha sido reportado en las tres encuestas nacionales $(1988 ; 0.11 \%$; 1993 ; $0.07 \% ; 1998,0.09 \%)$. Así se observa cómo la mariguana sigue siendo la principal droga consumida y los inhalables, que ocupaban el segundo lugar en 1988, fueron desplazados por la cocaína, cuyo aumento es notable. ${ }^{24}$ En adolescentes de 12 a 17 años de edad el consumo de cualquier droga alguna vez en la vida tuvo un aumento significativo que se elevó sobre todo entre 1993 y 1998.

La asistencia a la escuela se convierte en un factor protector importante para el consumo, si bien las diferencias en los índices de consumo entre estudiantes y no estudiantes son importantes sólo en los varones, esta relación ha sido observada también en estudios con menores trabajadores ${ }^{25} \mathrm{y}$ entre estudiantes ${ }^{4,17}$ cuando se comparan los índices de consumo entre aquellos que estudiaron el año anterior con los que no asistie- 
ron a la escuela, sugiriendo que aún perduran diferencias por género en el papel de la educación formal.

Una proporción importante, $24 \%$ de los varones y $20 \%$ de las mujeres, no consideran que sea peligroso usar drogas, si bien considerar esta conducta como peligrosa no limita el que los jóvenes decidan experimentar con drogas, sí previene que continúen usándolas después de haber experimentado los efectos, como lo hace la mayoría de los menores. Es por tanto importante aumentar la proporción de menores que considera que usar drogas es peligroso. Debe reforzarse la idea de que las drogas dañan la salud, ya que éste es el principal motivo que dan adolescentes para no acercarse a las drogas.

Uno de los factores que más consistentemente se ha asociado con el consumo de drogas es la disponibilidad; 18,26 cada vez más jóvenes experimentan con drogas por tenerlas disponibles en su medio, de hecho los datos de la encuesta señalaron que a 35\% de los adolescentes les era fácil obtener drogas y que el riesgo de experimentar con ellas se incrementaba 1.89 veces cuando esto ocurría.

El principal vector para el consumo de drogas son los amigos, ${ }^{27}$ con frecuencia usuarios experimentales que no han sufrido las consecuencias del abuso, son quienes se presentan como la mejor prueba de que el riesgo de consecuencias adversas no es tan elevado. Esto implica un reto importante para la política en esta materia, sólo en unos cuantos casos la droga fue proporcionada por vendedores y esto ocurrió únicamente en los varones. La manera como los jóvenes obtienen su droga a partir de la venta, obliga a unir medidas de control de la demanda con el control de la oferta mediante intervenciones oportunas que protejan al menor de ulterior dependencia de las drogas, problemas de salud y conductas antisociales y delictivas.

Los menores obtienen frecuentemente sustancias inhalables en las casas de familias; el uso extendido de los productos susceptibles de ocasionar abuso por sus efectos psicotrópicos es elevado, y por ello es importante cuidar la disponibilidad dentro del hogar y concienciar a los padres de familia con el fin de que proporcionen adecuada orientación a sus hijos, sin incitar su curiosidad y vigilar su comportamiento. Los lugares más comunes para la obtención de drogas son los espacios públicos en los que se requiere mayor vigilancia; como las discotecas son lugares propicios para la obtención de cocaína, es indispensable contar con la cooperación de sus dueños y administradores para evitar la distribución y consumo de ésta y otras sustancias.

La disponibilidad no se limita al acceso físico a la sustancia, sino también a la proporción de personas que la consume en el entorno inmediato del menor y a las normas sociales. ${ }^{26}$ Como pudo apreciarse, el hecho de que los padres vieran mal el uso de drogas no fue un factor que limitara la experimentación con drogas, pero sí fue una variable importante para que los menores abandonaran el consumo después de haber experimentado sus efectos, de ahí que es muy importante reforzar la poca tolerancia social ante el consumo que se observa todavía en el país.

Si bien las normas sociales de los padres no impidieron que adolescentes probaran drogas, la conducta de éstos fue especialmente importante. Cuando el padre, la madre o los hermanos usan drogas es muy probable que el o la adolescente experimente y continúe usándolas, de ahí que interese retroalimentar a los padres en este sentido.

Durante la adolescencia es más importante la opinión de los amigos que la de los padres ${ }^{27}$ cuando los amigos no reprueban el uso, o son indiferentes frente a este fenómeno, es más fácil que adolescentes prueben drogas; también es un hecho que perciban más aceptación entre los jóvenes en general que entre sus amigos en su entorno inmediato. Es por tanto importante incluir en las campañas de prevención información sobre la magnitud real del problema y las actitudes que prevalecen en nuestro medio, con el fin de evitar que una percepción inadecuada facilite la experimentación con drogas.

Fue interesante constatar que las razones más importantes que dan adolescentes para haber probado drogas son de naturaleza personal más que social; los varones querían saber si les gusta, y comprobar si se sentían bien, como les habían dicho; las razones sociales, esto es, usar drogas porque los amigos lo hacen, es menos común. Es importante profundizar en las motivaciones que llevan a adolescentes a probar drogas con el fin de ofrecer alternativas eficientes. Las mujeres querían saber también si las drogas las tranquilizaban. Estos datos, junto con la evidencia de los elevados índices de depresión, ideación y actos suicidas en los adolescentes de uno $u$ otro sexo, ${ }^{22}$ refuerzan la necesidad de desarrollar campañas que permitan detectar a los menores que tienen problemas emocionales, y darles la atención apropiada para evitar que consuman drogas como un mecanismo para enfrentar problemas de esta naturaleza.

Un factor para considerar en ulteriores estudios es la posible distinción entre los usuarios de diferentes drogas. Encuestas entre estudiantes, ${ }_{1}^{17}$ cuyos tamaños de muestra son generalmente mayores, que utilizan técnicas de regresión logística y que comparan a los estudiantes que incluían cada una de las drogas estudiadas con aquellos que usaban otras drogas, 
han documentado asociaciones entre características sociodemográficas y la elección de determinadas drogas. Así, se encontró que el ser varón aumentaba la probabilidad de usar drogas no médicas, mientras que en la mujer era más probable la utilización de drogas médicas; tener menos de 15 años y no trabajar o ser hijo de un jefe de familia con baja escolaridad predecía el uso de inhalables, mientras que lo opuesto aumentaba el riesgo de usar cocaína. Sin embargo, no se encontraron diferencias en variables del contexto (uso entre amigos, disponibilidad, percepción de riesgo y tolerancia social), y psicológicas (depresión e ideación suicida), similares a las utilizadas en este estudio, y la elección del tipo de droga con la excepción de la asociación entre mayor tolerancia social y uso de cocaína. Será importante determinar si estas variaciones se siguen presentando al haberse modificado el panorama del consumo en el país.

Finalmente, los resultados de este análisis indican que los factores incluidos en el estudio fueron apropiados para predecir la experimentación con drogas, pero fueron menos eficientes para entender el paso de la experimentación al uso continuado, y, menos aún, para explicar el uso problemático. Es por tanto necesario avanzar en el estudio de factores que permitan identificar a los menores con mayor probabilidad de desarrollar dependencia, quienes requieren intervenciones preventivas de mayor intensidad.

\section{Referencias}

1.C astro ME, Rojas E, García G, De la Serna J. Epidemiología del uso de drogas en la población estudiantil.Tendencias en los últimos 10 años. Salud Mental 1986; 9:80-85.

2. De la Serna J, Rojas E, Estrada MA, Medina-Mora ME. Medición del uso de drogas en estudiantes de educación media y media superior del Distrito Federal y zona conurbada, 1989. Memorias de la IV Reunión de Investigación. México, D.F:. Instituto Mexicano de Psiquiatría, 1991:183-187. 3. Medina-Mora ME, Rojas E, Juárez F, Berenzon S, C arreño S, G alván J et al. Consumo de sustancias con efectos psicotrópicos en la población estudiantil de enseñanza media y media superior de la República Mexicana. Salud Mental 1993;16: 2-8.

4.Villatoro J, Medina-Mora ME, C ardiel H, Fleiz C,Alcántara E, Hernández $\mathrm{S}$ et al. La situación del consumo de sustancias entre estudiantes de la Ciudad de México: medición otoño 1997. Salud Mental 1999; 22:18-30. 5. 0 rtiz A, Rodríguez E, G alván J, Soriano A, Flores JC . G rupo interinstitucional para el desarrollo del Sistema de Reporte de Información en Drogas. Resultados de la aplicación de la cédula: «Informe individual sobre consumo de drogas». Tendencias en el área metropolitana. México, D.F.: Instituto Mexicano de Psiquiatría, 1999.

6. Tapia-Conyer R, Kuri P, Cravioto P, Revuelta A. Sistema de Vigilancia Epidemiológica de las Adicciones (Sisvea). Informe. México, D.F.: Secretaría de Salud, 1998.

7. Centros de Integración Juvenil. El consumo de drogas ilícitas en México: población general, estudiantes, menores trabajadores, pacientes usuarios de drogas. Informe. México, D.F.: CIJ, 1999.
8. United $\mathrm{N}$ ations $\mathrm{O}$ ffice on D rug C ontrol and Crime Prevention. G lobal Illicit D rugTrends 2001. N ueva York (N Y):UN O D C CP, 1987.

9. Secretaría de Salud. Encuesta Nacional de Adicciones 1998. México, D.F.: SSA, 1999.

10. Merikangas KR, Mehta RL, Molnar BE, W alters EE, Swendsen JD, A guilar-G axiola SA et al. Comorbidity of substance use disorders with mood and anxiety disorders: Results of the International Consortium of Psychiatric Epidemiology. Addict Behav 23:893-907.

11. C astro ME. Manual para la utilización del cuestionario sobre el uso de drogas y problemas asociados, dirigido a muestras de estudiantes de enseñanza media y media superior. Reporte interno, México. D.F.: Instituto Mexicano de Psiquiatría, 1987

12. Medina-Mora ME, Gómez-Mont F, Campillo-Serrano C. Validity and reliability of a high school drug use questionnaire among Mexican students. Bull N arc 1981;33:67-76.

13. Smart R, Hughes P, Johnston LD, Medina-Mora ME.A methodology for student drug use surveys. Ginebra: W orld Health O rganization, 1980;W HO offset publication N 0.50.

14. Medina-Mora ME. Prevalencia del consumo de drogas en algunas ciudades de la República Mexicana. Encuestas de hogares. Enseñanza Invest Psicol 1981; 4:111-125.

15.Tapia-C onyer R, Medina-Mora ME, Sepúlveda J, D e la Fuente R, Kumate J. La Encuesta N acional de Adicciones de México. Salud Publica Mex 1990; 32:507-522.

16. Dirección General de Epidemio logía, Secretaría de Salud. Segunda Encuesta $N$ acional de Adicciones 1993. México, D.F.: SSA, 1994.

17. Medina-Mora ME,Villatoro J, López E, Berenzon S, Carreño S, Juárez F. Los factores que se relacionan con el inicio, el uso continuado y el abuso de sustancias psicoactivas en adolescentes mexicanos. Gac Med Mex 1995;131:383-387.

18.Villatoro J, Medina-Mora ME, Berenzon S, Juárez F, Rojas E, Carreño S. D rug use pathways among high school students of Mexico.Addiction 1998; 93:1557-1588.

19. Johnston L. Monitoring the future a continuing study of the lifestyles and values of youth. Ann Arbor (MI): Institute for Social Research, University of Michigan, 1989.

20. Roberts E. Reliability of the CES-D scale in different ethnic contexts. Psychiatry Res 1980; 2:125-134.

21. Mariño MC, Medina-Mora ME, Chaparro J, González-Forteza C. Confiabilidad y estructura factorial del CES-D en adolescentes mexicanos. Rev Mex Psicol 1993;10:141-145.

22. González-Forteza C, Mariño C, Rojas E, Mondragón L, Medina-Mora ME. Intento de suicidio en estudiantes de la ciudad de Pachuca, Hidalgo, y su relación con el malestar depresivo y el uso de sustancias. Rev Mex Psicol 1998; 15:165-175.

23. Berumen y asociados. Reporte metodológico de la Tercera Encuesta $\mathrm{N}$ acional de Adicciones, México, D.F.: casa ed. 1998.

24. Secretaría de Salud. Encuesta Nacional de Adicciones 1988. México, D.F.: SSA, 1990.

25. Medina-Mora ME, Villatoro J, Fleiz C. Uso indebido de sustancias. En: Estudio de niños, niñas y adolescentes entre 6 y 17 años.Trabajadores en 100 cuidades. México, D.F.: Sistema $\mathrm{N}$ acional para el Desarrollo Integral de la Familia/ Fondo de las N aciones Unidas para la Infancia, 1999: 369-374.

26.0 ffice of Technology Assessment for Understanding and Preventing. Substance abuse and addiction.W ashington, D.C :C Congress of the United States, 1994: 236.

27. Kellam S, W erthamer L, D olan L, Brown H, Mayer L, Rebok $G$ et al. Developmental epidemiologically based preventive trials: Baseline modeling of early target behaviors and depressive symptoms.Am J Community Psychol 1991; 19:563-584. 